\title{
Determinantes da Probabilidade de Subnotificação de Crimes Contra o Patrimônio no Brasil
}

\author{
Determinants of the Probability of Underreporting Property Crimes in Brazil
}

\author{
Adrielle Cléssia Martins ${ }^{a}$ \\ Evandro Camargos Teixeira ${ }^{\mathrm{b}}$ \\ Gercione Dionizio Silva ${ }^{c}$
}

\begin{abstract}
Resumo: O objetivo deste estudo é analisar os determinantes da probabilidade de subnotificação de crimes contra o patrimônio no Brasil. Para isso, modelou-se o processo de decisão da vítima em registrar ou não a vitimização à polícia, fundamentando-se na hipótese de racionalidade econômica através das informações da Pesquisa Nacional por Amostra de Domicílios (PNAD) de 2009. Para alcançar tal objetivo, foram estimados dois modelos Probit, um para furtos e outro para roubos, encontrando-se evidências de que algumas características socioeconômicas e demográficas da vítima, tais como idade, escolaridade, renda e região de residência determinam sua decisão de registrar ou não o crime.
\end{abstract}

Palavras-chave: Subnotificação; Crimes Patrimoniais; Brasil.

Classificação JEL: C25; K42; K49.

\begin{abstract}
The aim of this study is to analyze the determinants of the probability of underreporting crimes against property in Brazil. To this end, the victim's decision process was modeled on whether or not to register victimization with the police, based on the hypothesis of economic rationality through information from the 2009 National Household Sample Survey (PNAD) of 2009. To achieve this goal, two Probit models were estimated, one for thefts and the other for robberies, finding evidence that some socioeconomic and demographic characteristics of the victim, such as age, education, income and region of residence determine his decision to register the crime or not.
\end{abstract}

Keywords: Underreporting; Property Crimes; Brazil.

\footnotetext{
${ }^{\text {a }}$ Graduada em Ciências Econômicas pela Universidade Federal de Viçosa (UFV).

E-mail: adriellecmarttins@gmail.com

${ }^{\mathrm{b}}$ Professor Associado I do Departamento de Economia (DEE) da Universidade Federal de Viçosa (UFV). E-mail: evandro.teixeira@ufv.br

${ }^{\mathrm{c}}$ Doutor em Economia Aplicada pela Universidade Federal de Viçosa (UFV). E-mail: gercige@ gmail.com
} 


\section{Introdução}

A criminalidade se configura como um fenômeno capaz de impactar negativamente o desempenho socioeconômico brasileiro, causando forte redução do nível de bem-estar da população. Nesse sentido, a maioria dos trabalhos nacionais e internacionais se preocupou em avaliar como variáveis socioeconômicas afetam a criminalidade, tendo como destaque os trabalhos de Andrade e Lisboa (2000), Araújo Júnior e Fajnzylber (2001), Fajnzylber e Araújo Júnior (2001), Oliveira (2005) e Santos e Kassouf (2008). Porém, estes estudos têm encontrado uma dificuldade empírica, em especial, que está relacionada à taxa de subnotificação da criminalidade presente nos dados oficiais, que subestimam o número real de crimes ocorridos em dada região e/ou país em um determinado período de tempo.

Nesse contexto, Justus e Scorzafave (2014) destacam como principais consequências da subnotificação de crimes a distorção na alocação de recursos destinados à segurança pública; a impossibilidade de avaliação correta da eficiência das autoridades competentes; um maior nível de criminalidade, causado por uma menor quantidade de inquéritos policiais e, consequentemente, elevação da probabilidade de êxito dos indivíduos na atividade ilegal.

No entanto, mesmo diante da denotada relevância, relativamente poucos estudos objetivaram investigar empiricamente os fatores que determinam as taxas de subnotificação de crimes ou "cifra obscura" da criminalidade. Logo, o presente estudo, dentro de uma abordagem econômica, tem como objetivo central analisar quais fatores socioeconômicos e características individuais estão mais relacionados com o processo de decisão da vítima em reportar ou não o crime sofrido às autoridades competentes.

Com relação à literatura internacional referente ao tema, os estudos de Myers (1980) e Goldeberg e Nold (1980) sustentam a hipótese de que as vítimas agem racionalmente em sua decisão de registrar ou não um crime. Por isso, ao realizarem a queixa, tendem a avaliar custos e vantagens. De forma complementar, Craig (1987) utilizou um teste empírico através de um conjunto de informações referentes a um bairro exclusivo e demonstrou que a taxa real de crimes é diferente da reportada. Por fim, Duce et al. (2000) investigaram se variáveis de caráter socioeconômico que refletem o sentimento de segurança dos cidadãos - como idade, sexo, educação e outras - são correlacionadas com a probabilidade de denúncia de um delito.

Analisando-se o contexto brasileiro, observa-se uma escassez maior de estudos relacionados à temática de subnotificação de crimes, sendo algumas exceções encontradas nos trabalhos de Santos e Kassouf (2008), que examinaram a influência dos fatores socioeconômicos sobre a subnotificação de crimes contra a propriedade. Já os estudos de Scorzafave et al. (2011) e Madalozzo e Furtado (2011) estimaram modelos com variáveis dependentes limitadas a fim de verificar os determinantes da vitimização e subnotificação. 
O presente estudo busca dessa forma diferenciar-se dos anteriores encontrados na literatura nacional sobre vitimização em alguns aspectos, particularmente daquele realizado por Santos e Kassouf (2008), que mais se assemelha a este. Em primeiro lugar, diferentemente do referido trabalho, este estudo se propõe a analisar os dados do suplemento de vitimização da PNAD de 2009, por serem informações mais recentes. Em segundo lugar, serão considerados os crimes contra a propriedade referentes a furtos e roubos, diferentemente do trabalho supracitado, que somente analisa a subnotificação de roubos. Por fim, o presente estudo, ao utilizar os dados da PNAD, possui informações de abrangência nacional. Já o trabalho de Santos e Kassouf (2008) utiliza dados apenas dos municípios de São Paulo, Rio de Janeiro, Recife e Vitória.

Além da contribuição na literatura, almeja-se ainda, a partir dos resultados encontrados, demonstrar a importância de políticas públicas na área de segurança voltadas para conscientização das vítimas, no intuito de que haja diminuição da subestimação nas taxas reais de crimes. Como já ressaltado, a subnotificação pode afetar diretamente a eficiência na alocação de recursos em segurança pública e aumentar indiretamente a criminalidade.

Além dessa introdução, o presente estudo está dividido em mais cinco seções. Nas três próximas, são apresentados o referencial teórico, os dados utilizados e a estratégia empírica empregada no estudo, respectivamente. Os resultados obtidos são destacados na quinta seção, e na sexta são apresentadas as considerações finais.

\section{Referencial Teórico}

Conforme salientado, os determinantes da subnotificação de crimes são variados, pois estão relacionados diretamente com as decisões do indivíduo considerado racional ${ }^{1} \mathrm{e}$ que busca maximizar sua utilidade. Nessa perspectiva, Myers (1980), precursor das análises de subnotificação, afirma que a probabilidade de o indivíduo registrar ou não a vitimização está diretamente relacionada às suas características, ao crime e à utilidade esperada. A hipótese adotada pelo autor é que a decisão da vítima depende de variáveis quantificáveis, como por exemplo, aquelas que refletem as racionalizações da vítima $e x$ post ao crime. Além disso, as vítimas nem sempre possuem interesse em denunciar os crimes (MYERS, 1980).

Analisando-se a decisão de registro de um crime do ponto de vista econômico é importante considerar que a este ato estão associados custos e benefícios. Assim, um indivíduo apenas irá registrar um crime se a utilidade esperada for maior do que a de não registrar (SANTOS; KASSOUF, 2008).

Partindo desta abordagem, o custo pode ser compreendido como tempo alocado para efetivar o registro à polícia, assim como o tempo que poderia ser dispendido, caso

\footnotetext{
${ }^{1}$ Comportamento racional implica basicamente em maximização consistente de uma função de utilidade bem ordenada (BECKER, 1962).
} 
futuramente tivesse que ser realizado o reconhecimento do suspeito e para os depoimentos, nos casos em que o criminoso é capturado e reconhecido, que denota custo de oportunidade. Pode-se considerar também como custos implícitos a possibilidade de represálias pelo ofensor ou custos morais, associados a alguns tipos de crimes, como, por exemplo, agressões físicas.

No entanto, a vítima avalia também os benefícios esperados derivados de sua decisão. Esses benefícios variam desde a recuperação dos bens roubados ou furtados e/ou prejuízos materiais sofridos a captura e punição dos criminosos, particularmente quando existem atos de violência envolvidos.

Assim, conforme ilustrado na Figura 1, o indivíduo confronta duas possibilidades em caso de denúncia do crime: o ofensor ser ou não capturado. Em ambos os casos, supondo-se que o crime tenha gerado prejuízo financeiro para a vítima, dois tipos de situações podem ocorrer: a vítima ser compensada de todos os danos sofridos ou não.

Figura 1: O Problema da Decisão de Registro de Um Crime

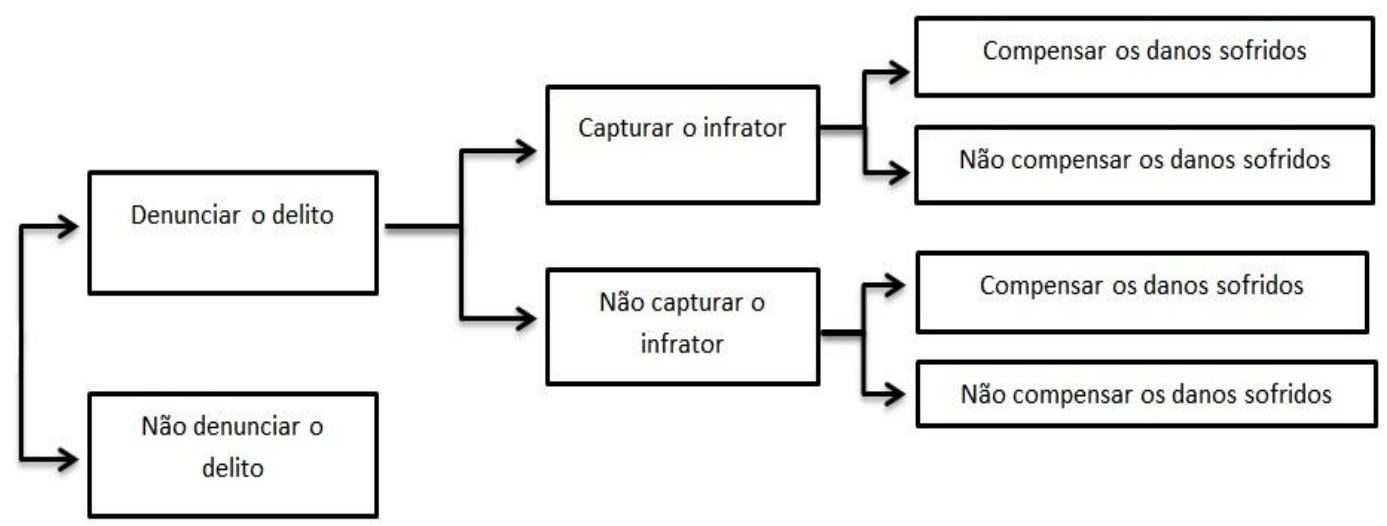

Fonte: Adaptada de Duce et. al. (2000).

Logo, a decisão de notificar e registrar um crime à polícia envolve avaliações e decisões das vítimas quanto a custos e benefícios esperados desta decisão. O grau de confiança da vítima na polícia e na justiça, a sensação de segurança e o grau de satisfação com a atuação das autoridades fazem com que aumente a probabilidade de o crime ser registrado às mesmas. Por outro lado, o estigma social, relacionado principalmente a alguns tipos específicos de ocorrências criminais, como por exemplo, nos casos de estupros e agressões, implica menor probabilidade de registro de um crime.

A utilidade esperada em ambas as situações (registrar ou não a ocorrência de um crime) pode ser expressa como:

$$
U_{i}=f_{i}\left(Z_{i}\right)+e_{i}
$$


em que: $U_{i}$ representa a utilidade esperada do indivíduo; $f_{i}\left(Z_{i}\right)$, uma função de utilidade determinada pelo vetor de variáveis, $Z_{i}$, associadas às escolhas do indivíduo; $e_{i}$, termo de erro da relação. O subscrito $i$ representa as possíveis ações do agente vitimado, podendo assumir dois valores: 0 , caso notifique o crime; 1 , caso contrário.

Myers (1980) define a população de indivíduos vitimados por um crime por $\Omega$, que subdivide-se entre aqueles que registraram a vitimização, $\Omega_{1}$, e aqueles que não registraram, $\Omega_{0}$. Levando-se em consideração que a decisão da vítima em registrar a vitimização é orientada pelo desejo de maximização da utilidade, se $X$ é um vetor de características da vítima e do crime, a utilidade de registro da vitimização $\left(U_{1}\right)$ será $U_{1}=$ $f_{1}(X)$; e a utilidade de não registrar $\left(U_{0}\right)$ será $U_{0}=f_{0}(X)$.

A partir da equação (1) e com base no pressuposto de racionalidade do indivíduo, a população denotada por $\Omega_{1}$, composta pelas vítimas que consideraram $U_{1} \geq U_{0}$, registra o crime. Pelo contrário, quando $U_{0}>U_{1}$, caso da população representada por $\Omega_{0}$, não haverá notificação do crime. Assim, tem-se um modelo de escolha binária, baseado na decisão tomada pela vítima de registrar (Registro $=1$ ) ou não (Registro=0). Todavia, deve-se observar que a utilidade associada ao registro ou não do crime não é diretamente observada, mas é indiretamente inferida pela decisão da vítima.

Em suma, observa-se que a vítima de um crime irá reportá-lo apenas se a utilidade esperada da decisão de reportar o crime for maior do que a utilidade esperada em não fazê-lo. Por sua vez, tal decisão será influenciada pelas características do crime e do indivíduo, do grau de confiança na eficiência da polícia, das perdas materiais e do medo de uma possível retaliação. Portanto, a subnotificação será resultado da decisão racional do indivíduo, pautada na relação entre a utilidade esperada em não reportar o crime vis- $a$ vis à utilidade esperada de se reportar.

\section{Dados}

A base de dados utilizada tem como fonte os microdados disponibilizados pela pesquisa suplementar de 2009 da Pesquisa Nacional por Amostra de Domicílios (PNAD), que é realizada anualmente desde 1967 pelo Instituto Brasileiro de Geografia e Estatística (IBGE). São obtidas dessa pesquisa informações socioeconômicas dos indivíduos e características dos domicílios. Além dessas variáveis, a PNAD realiza ainda questionários suplementares, sendo que a de 2009 possui questionários relacionados à "Vitimização e Justiça". No entanto, não são especificadas informações adicionais, tais como gastos com segurança, quantidade de delegacias, celeridade do sistema judiciário, ou outras informações similares. A despeito destas limitações, contudo, esta é a única pesquisa amostral de larga escala que permite monitorar se o entrevistado sofreu algum tipo de crime patrimonial e se procurou a polícia.

Nessa pesquisa, foram entrevistadas 399.387 pessoas em 153.387 domicílios. Entretanto, foram realizados alguns filtros para se chegar à amostra apropriada para o 
estudo. Foram consideradas apenas vítimas de crimes patrimoniais com idade maior ou igual a 18 anos e foram excluídas aquelas que se autodeclararam indígenas ou amarelas ${ }^{2}$.

Além disso, nas estimações realizadas considera-se a característica do plano amostral da PNAD (amostra complexa). Notadamente, esta deve ser considerada, visto que a existência de diferentes estratificações e conglomerados levam a distintas probabilidades de seleção entre os indivíduos analisados.

\section{Metodologia}

Como fora destacado, a vítima de um crime, visando maximizar sua utilidade, deve escolher entre relatar o crime ou não. Desse modo, para elucidar quais fatores socioeconômicos mais se relacionam com a probabilidade de subnotificação da vitimização, optou-se pela utilização de um modelo com variável dependente binária.

Mediante o objetivo de se verificar quais variáveis mais se relacionam à subnotificação de furtos e roubos, serão estimados dois modelos Probit. Notadamente, deve-se observar que estas são modalidades distintas de crimes. No roubo, ocorre a subtração de qualquer objeto com ameaça, violência ou uso de força. Por outro lado, o furto ocorre sem o uso de ameaça ou violência, de modo que seja comum a não percepção do crime pela vítima no momento em que este ocorre. Assim, objetiva-se, através destas estimações, determinar quais as características dos vitimados influenciam a probabilidade de estes notificarem as autoridades os referidos crimes.

Além disso, no modelo de probabilidade binária utilizado (Probit), são consideradas a magnitudes das variáveis ao se estimar os coeficientes. Logo, o efeito parcial de qualquer variável deixa de ser constante. As probabilidades estimadas por este método serão consistentes com as leis da probabilidade, ou seja, estarão contidas no intervalo entre $[0,1]$.

O modelo Probit pode ser apresentado da seguinte forma:

$$
P(y=1 \mid x)=P\left(y=1 \mid x_{1}, x_{2}, \ldots, x_{k}\right)
$$

Em que x representa o conjunto de variáveis explicativas e y é a variável dependente.

Pode-se ainda apresentar uma classe de modelos de resposta binária como:

$$
P(y=1 \mid x)=G\left(\beta_{0}+\beta_{1} x_{1}+\beta_{2} x_{2}+\cdots+\beta_{k} x_{k}\right)
$$

em que a função $G$ assume valores estritamente entre 0 e $1(0<\mathrm{G}(z)<1)$, para todos os números $z$ reais. Trata-se de uma condição necessária para que as probabilidades estimadas estejam entre 0 e 1.

\footnotetext{
${ }^{2}$ Tal exclusão se justifica pelo fato de amarelos e indígenas corresponderem a um pequeno número de observações.
} 
Nesse sentido, a fim de garantir que as probabilidades para a função $G$ estejam entre 0 e 1, várias funções não lineares têm sido sugeridas. Uma delas é a função de distribuição cumulativa (f.d.c.) normal padrão, que é a função $G$ no modelo Probit. Expressa-se esta função da seguinte forma:

$$
G(z)=\Phi(z) \equiv \int_{-\infty}^{z} \phi(v) d v, \quad \text { onde } \phi(z)=(2 \pi)^{-1 / 2} \exp \left(-\frac{z^{2}}{2}\right)
$$

A escolha de $G$ assegura que (3) assuma valores entre 0 e 1 em todos os parâmetros. Por sua vez, para determinar o efeito parcial das variáveis explicativas, é necessário utilizar o cálculo da derivação parcial. Assim, sendo G uma f.d.c. de uma variável aleatória contínua, $g$ é uma função de densidade de probabilidade e $x_{j}$ um vetor de variáveis aproximadamente contínuo, sendo seu efeito parcial obtido da seguinte forma:

$$
\frac{\partial p(x)}{\partial x_{j}}=g\left(\beta_{0}+x \beta\right) \beta_{j}, \quad \text { onde } g(z) \equiv \frac{d G}{d z}(z)
$$

Ademais, seja $x_{1}$ uma variável explicativa binária, o efeito parcial de se alterar $x_{1}$ de 0 para 1 , dadas as demais variáveis, será:

$$
G\left(\beta_{0}+\beta_{1}+\beta_{2} x_{2}+\cdots+\beta_{k} x_{k}\right)-G\left(\beta_{0}+\beta_{2} x_{2}+\cdots+\beta_{k} x_{k}\right)
$$

Pelo exposto, com a utilização dos modelos de resposta binária, busca-se explicar os efeitos das variáveis incluídas no modelo sobre a probabilidade de resposta $P(y=$ $1 \mid x)$. Além disso, ambas as especificações estimadas têm como base a equação (3), sendo as variáveis dependentes a representação da decisão da vítima de não registrar o furto (NREGISTROFURTO) e não registrar o roubo (NREGISTROROUBO). Mais especificamente, o indivíduo é questionado se registrou o último furto ou roubo em alguma delegacia de polícia, incluindo delegacias virtuais. Logo, estas variáveis são determinadas da seguinte forma:

$$
\begin{aligned}
& \text { NREGISTROFURTO }=\left\{\begin{array}{c}
1 \text { se o furto não foi registrado; } \\
0 \text { caso contrário. }
\end{array}\right. \\
& \text { NREGISTROROUBO }=\left\{\begin{array}{c}
1 \text { se o roubo não foi registrado; } \\
0 \text { caso contrário. }
\end{array}\right.
\end{aligned}
$$

Por sua vez, as variáveis explicativas, bem como seus sinais esperados, são apresentadas no Quadro 1. 


\section{Quadro 1 - Variáveis Explicativas a Serem Utilizadas na Estimação do Modelo Econométrico}

\begin{tabular}{|c|c|c|}
\hline Variáveis & Descrição & Sinal esperado $^{1}$ \\
\hline Sexo & $\begin{array}{l}\text { Dummy que recebe valor igual a } 1 \text { se a vítima é do sexo } \\
\text { masculino, e } 0 \text { caso seja do sexo feminino. }\end{array}$ & SSE \\
\hline Idade & Idade das vítimas $\left(\right.$ idade $\left._{i}\right)$. & Negativo \\
\hline Escolaridade & $\begin{array}{l}\text { Vetor de variáveis dummies que mensuram o nível de } \\
\text { escolaridade das vítimas a partir dos anos de estudo: } 1 \\
\text { ano ou menos }\left(\text { analfabeto }_{i}\right) ; 2 \text { a } 5 \text { anos }\left(\text { basico }_{i}\right) ; 5 \text { a } 9 \\
\text { anos }\left(\text { fundamental }_{i}\right) ; 9 \text { a } 11 \text { anos }\left(\text { medio }_{i}\right) ; 11 \text { anos ou } \\
\text { mais }\left(\text { superior }_{i}\right) \text {. }\end{array}$ & SSE \\
\hline Ocupação & $\begin{array}{l}\text { Dummy que recebe valor igual a } 1 \text { se a vítima trabalha, e } \\
0 \text { caso contrário. }\end{array}$ & Positivo \\
\hline Renda & $\begin{array}{l}\text { Logaritmo da renda domiciliar per capita em } 100 \text { reais } \\
\text { das vítimas. }\end{array}$ & Negativo \\
\hline Estado Civil & $\begin{array}{l}\text { Dummy que recebe valor igual } 1 \text { se a vítima for casada, e } \\
0 \text { caso contrário. }\end{array}$ & SSE \\
\hline Cor & $\begin{array}{l}\text { Dummy que recebe valor igual a } 1 \text { se a vítima for branca, } \\
\text { e } 0 \text { caso contrário. }\end{array}$ & SSE \\
\hline $\begin{array}{l}\text { Região } \\
\text { Censitária }\end{array}$ & $\begin{array}{l}\text { Dummy que recebe valor igual a } 1 \text { se a vítima reside em } \\
\text { área urbana, e } 0 \text { na área rural. }\end{array}$ & SSE \\
\hline $\begin{array}{l}\text { Regiões } \\
\text { Brasileiras }\end{array}$ & $\begin{array}{l}\text { Dummies para as regiões brasileiras onde as vítimas } \\
\text { residem, sendo o } \mathrm{NO} \text { a referência, portanto, ausente no } \\
\text { modelo. }\left(N O_{i}, N E_{i}, C O_{i}, S E_{i} \text { e } S U_{i}\right) \text {. }\end{array}$ & SSE \\
\hline Perdas & $\begin{array}{l}\text { As variáveis } \text { Perdasroubo }_{i} \text { e } \text { Perdasfurto }_{i} \text { levam em } \\
\text { consideração a ocorrência de roubo/furto de carro ou } \\
\text { motocicleta como proxies para elevados prejuízos } \\
\text { incorridos pelas vítimas. }\end{array}$ & Negativo \\
\hline
\end{tabular}

Fonte: Elaboração própria.

Nota: ( $\left.{ }^{1}\right)$ SSE $=$ Sem sinal esperado.

Conforme apresentado no Quadro 1, espera-se que as variáveis Idade, Renda e Perdas sejam negativamente relacionadas ao não registro do último furto ou roubo. 
Segundo Duce et. al (2000), quanto maior a idade do indivíduo, mais elevada sua probabilidade de registrar um crime às autoridades. Para Santos e Kassouf (2008), maiores níveis de renda se relacionam com maiores perdas, o que levaria à probabilidade mais elevada do crime ser reportado. Análise similar é realizada pelos autores para o caso da variável Perda.

Por outro lado, tal qual apresentado por Santos e Kassouf (2008), os indivíduos que trabalham estão sujeitos a maiores custos em termos de tempo e, assim, custos de oportunidade mais elevados de notificar um crime às autoridades. Diante disso, é esperada relação positiva entre os indivíduos estarem ocupados e a probabilidade de não registrar o crime.

Por fim, não há a priori sinais esperados para as variáveis sexo, escolaridade, estado civil, cor, região censitária e aquelas que denotam as regiões brasileiras. Nesse sentido, espera-se que não haja diferença comportamental entre homens e mulheres quanto ao registro de um crime de furto ou roubo (SANTOS; KASSOUF, 2008).

Quanto à escolaridade, há na literatura uma contradição quanto ao sinal esperado. Segundo Goldeberg e Nold (1980), a probabilidade de se registrar um crime está positivamente correlacionada ao nível de escolaridade dos indivíduos. Porém, para Santos e Kassouf (2008), o custo de denunciar um crime para indivíduos com elevada escolaridade será maior. Este, por sua vez, implicará em relação negativa entre escolaridade e probabilidade dos indivíduos reportarem crimes.

Santos e Kassouf (2008) afirmam ainda que indivíduos que vivem conjugalmente podem ser influenciados de forma positiva ou negativa na sua decisão de reportar a vitimização. Tal influência vai depender do comportamento do (a) parceiro (a), além do seu poder de influência sobre o comportamento do cônjuge vitimado. Por isso, a princípio não pode ser estabelecida nenhuma relação entre estado civil da vítima e a probabilidade de não registro do crime.

Por fim, a partir da estimação do modelo econométrico, será realizado um teste de ajustamento, com a estimação da Curva ROC (Receiver Operating Characteristic). A curva ROC é baseada em dois conceitos: sensibilidade e especificidade. A sensibilidade é definida como a probabilidade de o resultado de um evento sob observação ter diagnóstico positivo, dado que o evento realmente é positivo, ou ainda fração de verdadeiros positivos. Por sua vez, a especificidade é definida como a probabilidade de o resultado de um evento sob observação ter diagnóstico negativo, dado que o evento é realmente negativo, ou fração de verdadeiro negativo.

Dessa forma, o princípio desta curva é representar graficamente a relação entre os verdadeiros positivos (sensibilidade) e os falsos positivos (especificidade) (BRAGA, 2000; CAMERON e TRIVEDI, 2010; FÁVERO e BELFIORE, 2014). Assim, a curva representa os pares de probabilidades estimadas dos verdadeiros positivos (sensibilidade, representada no eixo vertical) e dos falsos positivos (dada pela diferença "1 especificidade", representada no eixo horizontal). Mais especificamente, a área sob a 
curva mede a capacidade de discriminação do modelo, ou seja, quanto mais próxima a curva estiver do ponto $(1,0)$, mais ajustado é o mesmo.

\section{Resultados e Discussão}

\subsection{Caracterização da Subnotificação de Crimes no Brasil}

$\mathrm{Na}$ seção anterior, foram apresentadas as relações esperadas entre a subnotificação dos crimes contra o patrimônio considerados no estudo, mediante as variáveis socioeconômicas incluídas. Para corroborar com os pressupostos iniciais, são retratadas algumas estatísticas descritivas na Tabela 1. Nesta, é possível observar relação entre características socioeconômicas dos indivíduos com o registro de crimes contra o patrimônio considerados no estudo, furtos e roubos, em 2009.

Conforme a referida Tabela, a quantidade de crimes não notificados de roubos é superior à de furtos. Tal resultado pode ser explicado pelas características das pesquisas de vitimização aliadas à forma pela qual os questionamentos são realizados. Goudriaan e Assen (2006), assim como Santos e Kassouf (2008), destacam que há algumas desvantagens na utilização das pesquisas de vitimização, quando comparadas às estatísticas oficiais: i) falta de memória do entrevistado quando questionado a respeito de ter sofrido um determinado crime a algum tempo, ainda mais quando a pergunta se refere ao último episódio vivenciado; ii) a realização da pesquisa em domicílios, de modo que a pessoa de referência responde pelos outros; iii) e o fato de não existir uma pesquisa com periodicidade e método de amostragem definidos, impedindo comparações. Segundo Reichel e Albanese (2014), há ainda a possibilidade de a informação dada pelo entrevistado ser falsa, como nos casos em que a vítima conhece o criminoso e prefere não declarar o crime.

Com relação às demais informações constantes na Tabela 1 , a diferença entre o percentual de homens e mulheres que efetivamente registraram os crimes de furtos ou roubos é pequena. Em média, a diferença entre os crimes de furto não registrados entre mulheres e homens é de 2,4 pontos percentuais. Já em relação aos roubos, a diferença é de apenas 1,7 pontos. De modo similar, observa-se que indivíduos não brancos apresentaram maior percentual de subnotificação, comparados a indivíduos brancos. Nesse sentido, em média, não brancos apresentam 2,5 pontos percentuais a mais de subnotificação de crimes.

Destaca-se, ainda, que para ambos os crimes analisados (furtos e roubos), a subnotificação dos crimes sofridos por indivíduos solteiros ou casados, que trabalham ou que moram em regiões urbanas, manteve-se relativamente baixa. Dito de outra forma, aproximadamente $10 \%$ dos indivíduos com alguma dessas características não notificaram os crimes considerados no estudo. Em contrapartida, a subnotificação dos crimes supracitados é maior para indivíduos desempregados (14,3\% para furtos e 8,5\% para roubos). 
Dentre as características apresentadas na Tabela 1, a escolaridade apresentou maior discrepância entre seus níveis em 2009. Em relação aos furtos, neste período, aproximadamente $20,2 \%$ dos indivíduos analfabetos não registram o crime sofrido. Por outro lado, a subnotificação do crime entre indivíduos com nível superior foi de 6,4\%. Embora a diferença entre a subnotificação do crime de roubo entre indivíduos analfabetos e com nível superior seja menor, há ainda substancial distinção entre os percentuais. Em síntese, a subnotificação entre indivíduos analfabetos é 7 pontos percentuais mais elevada.

\section{Tabela 1 - Percentual de Furtos e roubos não registrados, de acordo com características socioeconômicas e demográficas das vítimas}

\begin{tabular}{|c|c|c|c|c|c|c|c|c|c|c|c|}
\hline \multirow{3}{*}{ Variável } & \multirow{3}{*}{ Categoria } & \multirow{2}{*}{\multicolumn{5}{|c|}{$\begin{array}{c}\text { Furtos } \\
\text { Subnotificacão }\end{array}$}} & \multicolumn{5}{|c|}{ Roubos } \\
\hline & & & & & & & \multicolumn{5}{|c|}{ Subnotificação } \\
\hline & & $\%$ & $\mathbf{N}$ & $\begin{array}{c}\text { Total } \\
\text { de obs. }\end{array}$ & Média & $\begin{array}{c}\text { Desvio } \\
\text { Padrão }\end{array}$ & $\%$ & $\mathbf{N}$ & $\begin{array}{l}\text { Total } \\
\text { de obs. }\end{array}$ & Média & $\begin{array}{l}\text { Desvio } \\
\text { Padrão }\end{array}$ \\
\hline \multirow{2}{*}{ Sexo } & Mulher & $11,50 \%$ & 241 & 2092 & \multirow{2}{*}{0,57} & \multirow{2}{*}{0,49} & $9,80 \%$ & 273 & 2800 & \multirow{2}{*}{0,53} & \multirow{2}{*}{0,49} \\
\hline & Homem & $9,10 \%$ & 244 & 2680 & & & $8,10 \%$ & 285 & 3500 & & \\
\hline \multirow{2}{*}{ Cor } & Não Branco & $11,50 \%$ & 261 & 2264 & \multirow{2}{*}{0,48} & \multirow{2}{*}{0,5} & $10 \%$ & 330 & 3306 & \multirow{2}{*}{0,43} & \multirow{2}{*}{0,49} \\
\hline & Branco & $8,90 \%$ & 224 & 2508 & & & $7,60 \%$ & 228 & 2994 & & \\
\hline \multirow{2}{*}{ Estado Civil } & Solteiro & $10,70 \%$ & 269 & 2537 & \multirow{2}{*}{0,45} & \multirow{2}{*}{0,49} & $9,70 \%$ & 355 & 3676 & \multirow{2}{*}{0,37} & \multirow{2}{*}{0,48} \\
\hline & Casado & $9,70 \%$ & 216 & 2235 & & & $7,70 \%$ & 203 & 2624 & & \\
\hline \multirow{2}{*}{$\begin{array}{c}\text { Região } \\
\text { Censitária }\end{array}$} & Rural & $12,60 \%$ & 39 & 310 & \multirow{2}{*}{0,9} & \multirow{2}{*}{0,28} & $8,70 \%$ & 22 & 252 & \multirow{2}{*}{0,94} & \multirow{2}{*}{0,23} \\
\hline & Urbana & $10 \%$ & 446 & 4462 & & & $8,90 \%$ & 536 & 6048 & & \\
\hline \multirow{2}{*}{ Ocupação } & Não trabalha & $14,30 \%$ & 36 & 251 & \multirow{2}{*}{0,91} & \multirow{2}{*}{0,28} & $8,50 \%$ & 41 & 484 & \multirow{2}{*}{0,91} & \multirow{2}{*}{0,28} \\
\hline & Trabalha & $9,30 \%$ & 344 & 3681 & & & $8,50 \%$ & 417 & 4889 & & \\
\hline \multirow{5}{*}{ Escolaridade } & Analfabeto & $20,20 \%$ & 54 & 267 & & & $13,60 \%$ & 33 & 243 & & \\
\hline & Básico & $14,60 \%$ & 103 & 705 & & & $11,70 \%$ & 78 & 668 & & \\
\hline & Fundamental & $11,30 \%$ & 109 & 962 & 9,11 & 4,44 & $8,20 \%$ & 97 & 1181 & 10,39 & 3,98 \\
\hline & Médio & $8,70 \%$ & 142 & 1639 & & & $9,30 \%$ & 248 & 2661 & & \\
\hline & Superior & $6,40 \%$ & 77 & 1199 & & & $6,60 \%$ & 102 & 1547 & & \\
\hline & $\mathrm{NO}$ & $9,30 \%$ & 61 & 654 & 0,75 & 0,26 & $7,10 \%$ & 74 & 1048 & 0,09 & 0,28 \\
\hline & $\mathrm{NE}$ & $14,20 \%$ & 143 & 1009 & 0,29 & 0,45 & $12,30 \%$ & 256 & 2084 & 0,38 & 0,49 \\
\hline Regiões & $\mathrm{CO}$ & $12,60 \%$ & 95 & 756 & 0,14 & 0,35 & $7,90 \%$ & 55 & 694 & 0,08 & 0,28 \\
\hline & SE & $8 \%$ & 113 & 1411 & 0,33 & 0,47 & $7,30 \%$ & 120 & 1633 & 0,35 & 0,47 \\
\hline & SU & $7,40 \%$ & 70 & 942 & 0,14 & 0,35 & $6,30 \%$ & 53 & 841 & 0,08 & 0,27 \\
\hline
\end{tabular}

Fonte: Elaborado pelos autores a partir dos dados do IBGE (2009).

Nota: \% (porcentagem), N (número de observações) e Média (média da variável).

Como a variável de escolaridade é extremamente importante, a Figura 2 foi construída com o intuito de reforçar o resultado verificado na Tabela 1. No geral, 
percebe-se que quanto menor o nível de escolaridade, mais elevada é a subnotificação de crimes contra o patrimônio.

Outro fator muito importante relacionado a subnotificação de furtos e roubos é o nível de renda domiciliar per capita das vítimas, vide Figuras 3 e 4, que é apresentada em decis. Levando-se em consideração que o grupo 1 corresponde aos $10 \%$ das vítimas mais pobres e o grupo 10 representa as $10 \%$ mais ricas, percebe-se a existência de relação inversamente proporcional entre renda domiciliar per capita e subnotificação. Para ilustrar essa relação de forma mais clara, na Figura 3, verifica-se que entre os $10 \%$ de vítimas mais pobres da amostra, cerca de $19,6 \%$ não registraram o furto, contra $4,8 \%$ entre os $10 \%$ mais ricos. Por sua vez, na Figura 4, observa-se que 13,8\% das vítimas mais pobres não notificaram o roubo, enquanto no grupo das $10 \%$ mais ricas esse percentual é reduzido para $4,8 \%$.

Figura 2: Percentual de Subnotificação de Furtos e Roubos por Nível de Escolaridade

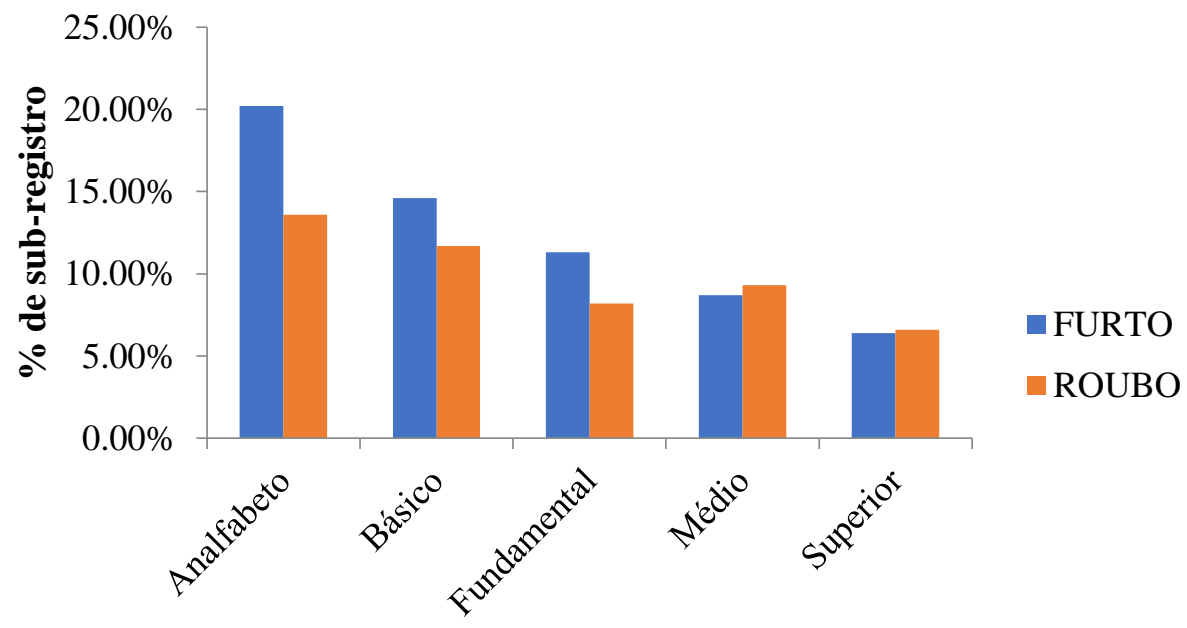

Escolaridade

Fonte: Elaboração própria a partir dos dados do IBGE (2009).

Figura 3: Percentual de Subnotificação de Furtos Correlacionado ao Nível de Renda Domiciliar Per Capita 


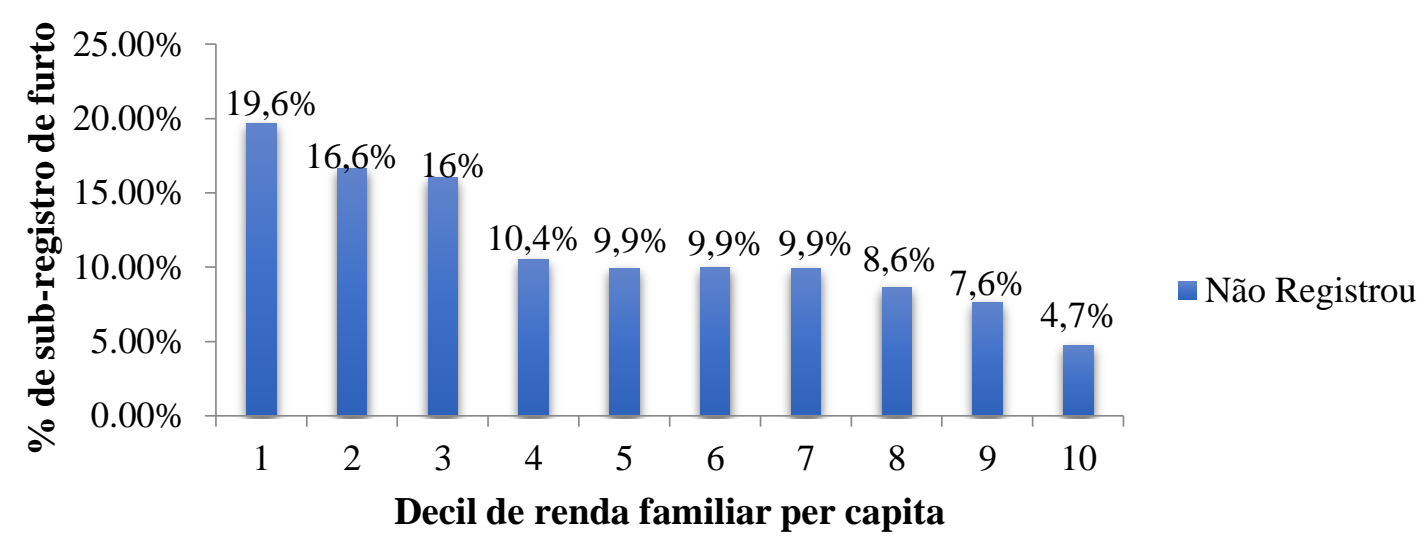

Fonte: Elaboração própria a partir dos dados do IBGE (2009).

A questão regional também se relaciona com a probabilidade de subnotificação das vítimas, o que também é abordado na referida Tabela 1. Verifica-se que os resultados para as duas modalidades de crime consideradas no estudo em relação à região censitária são destoantes. Assim, a subnotificação de furtos é mais elevada na zona rural e, em contrapartida, a subnotificação de roubos é ligeiramente superior no meio urbano.

No que tange à subnotificação entre as regiões brasileiras, verifica-se que a região Sul foi aquela com menor percentual de crimes não registrados. Notadamente, apenas $7,4 \%$ dos furtos e $6,3 \%$ dos roubos não foram registrados. Em contrapartida, a região Nordeste apresentou maior contingente de subnotificação, sendo 14,2\% de furtos e 12,3\% de roubos não registrados.

\section{Figura 4: Percentual de Subnotificação de Roubos Correlacionado ao Nível de Renda Domiciliar Per Capita}




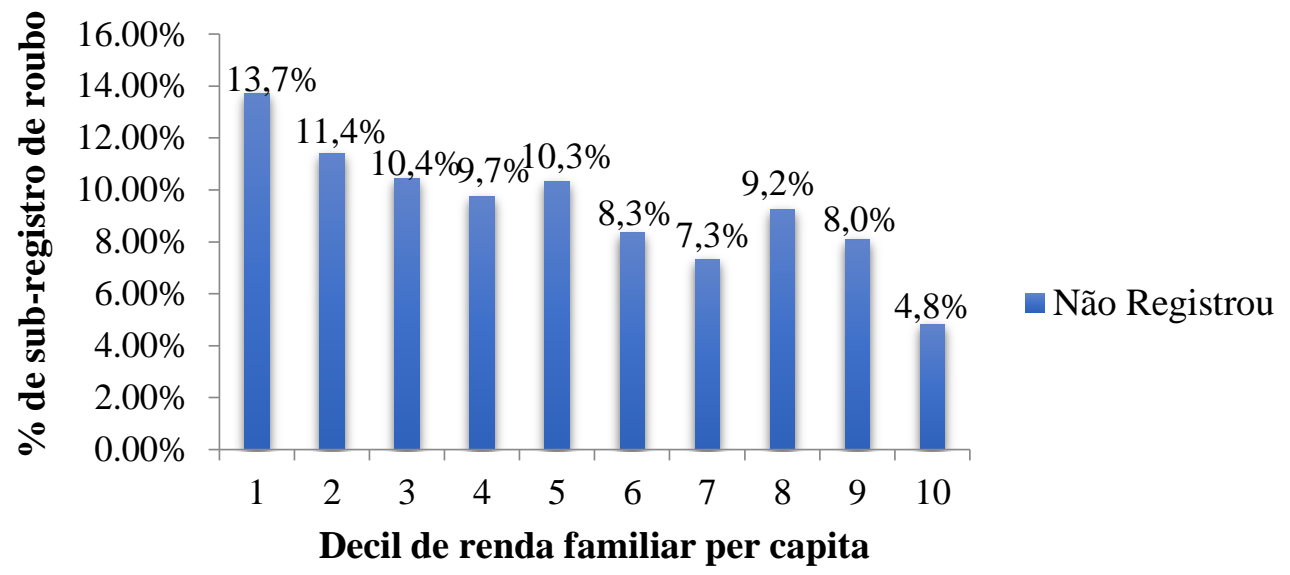

Fonte: Elaboração própria a partir dos dados do IBGE (2009).

Após avaliação das subnotificações por regiões brasileiras, a análise é desagregada por estados. Logo, na Tabela 2, são apresentadas as porcentagens de subnotificação de furtos e roubos por unidades federativas brasileiras em 2009. Observase que Alagoas é o estado que apresenta maior incidência de subnotificação de furtos no Brasil, seguido por Paraíba, Sergipe, Maranhão, Mato Grosso, Goiás, Ceará e Pará. Por sua vez, Alagoas, Maranhão, Sergipe, Espírito Santo, Ceará e Tocantins destacam-se pelas maiores taxas de subnotificação de roubos. Além disso, percebe-se que Amazonas e Distrito Federal apresentam menores taxas de subnotificação de furtos e roubos, respectivamente. Como esperado mediante a análise por regiões brasileiras, os estados da região Nordeste lideram o ranking de subnotificação de furtos e roubos no período analisado. 
Tabela 2 - Percentual de furtos e roubos não registrados por estados brasileiros

\begin{tabular}{|c|c|c|c|c|c|c|}
\hline \multirow{2}{*}{ Estado / Estatísticas } & \multicolumn{3}{|c|}{ Sub-registro Furto } & \multicolumn{3}{|c|}{ Sub-registro Roubo } \\
\hline & $\mathbf{N}$ & $\%$ & Total de obs. & $\mathbf{N}$ & $\%$ & Total de obs. \\
\hline Rondônia & 7 & $7,45 \%$ & 94 & 4 & $4,40 \%$ & 91 \\
\hline Acre & 8 & $12,31 \%$ & 65 & 6 & $10,53 \%$ & 57 \\
\hline Amazonas & 2 & $2,90 \%$ & 69 & 6 & $5,66 \%$ & 106 \\
\hline Roraima & 3 & $5,56 \%$ & 54 & 2 & $7,69 \%$ & 26 \\
\hline Pará & 22 & $15,60 \%$ & 141 & 36 & $6,91 \%$ & 521 \\
\hline Amapá & 3 & $6,67 \%$ & 45 & 2 & $4,55 \%$ & 44 \\
\hline Tocantins & 4 & $5,48 \%$ & 73 & 5 & $14,29 \%$ & 35 \\
\hline Maranhão & 13 & $23,21 \%$ & 56 & 16 & $20,51 \%$ & 78 \\
\hline Piauí & 6 & $13,64 \%$ & 44 & 3 & $5,77 \%$ & 52 \\
\hline Pará & 34 & $16,75 \%$ & 203 & 81 & $17,57 \%$ & 461 \\
\hline Rio Grande do Norte & 8 & $13,79 \%$ & 58 & 9 & $8,26 \%$ & 109 \\
\hline Paraíba & 8 & $24,24 \%$ & 33 & 9 & $11,39 \%$ & 79 \\
\hline Pernambuco & 10 & $8,33 \%$ & 120 & 24 & $6,54 \%$ & 367 \\
\hline Alagoas & 7 & $30,43 \%$ & 23 & 10 & $21,28 \%$ & 41 \\
\hline Sergipe & 13 & $22,81 \%$ & 57 & 11 & $18,64 \%$ & 59 \\
\hline Bahia & 17 & $7,46 \%$ & 228 & 43 & $8,16 \%$ & 527 \\
\hline Minas Gerais & 28 & $6,26 \%$ & 447 & 24 & $6,56 \%$ & 366 \\
\hline Espírito Santo & 9 & $12,16 \%$ & 74 & 12 & $18,18 \%$ & 66 \\
\hline Rio de Janeiro & 9 & $6,87 \%$ & 131 & 18 & $5,50 \%$ & 327 \\
\hline São Paulo & 38 & $7,74 \%$ & 491 & 43 & $7,12 \%$ & 604 \\
\hline Paraná & 19 & $7,36 \%$ & 258 & 22 & $9,24 \%$ & 238 \\
\hline Santa Catarina & 15 & $10,07 \%$ & 149 & 2 & $3,85 \%$ & 52 \\
\hline Rio Grande do Sul & 19 & $5,18 \%$ & 367 & 19 & $4,48 \%$ & 424 \\
\hline Mato Grosso do Sul & 7 & $6,60 \%$ & 106 & 6 & $8,45 \%$ & 71 \\
\hline Mato Grosso & 18 & $17,31 \%$ & 104 & 9 & $10,11 \%$ & 89 \\
\hline Goiás & 45 & $17,24 \%$ & 261 & 28 & $12,23 \%$ & 229 \\
\hline Distrito Federal & 8 & $4,73 \%$ & 169 & 6 & $2,68 \%$ & 224 \\
\hline Média & \multicolumn{3}{|c|}{33,41} & \multicolumn{3}{|c|}{30,73} \\
\hline Desvio Padrão & \multicolumn{3}{|c|}{10,75} & \multicolumn{3}{|c|}{9,68} \\
\hline
\end{tabular}

Fonte: Elaboração própria a partir dos dados do IBGE (2009).

Nota: N (número de observações que não registraram o crime) e \% (porcentagem).

Apesar de úteis para a interpretação dos resultados do presente estudo, as estatísticas descritivas apresentadas não podem sustentar as hipóteses de causalidade entre as variáveis explicativas e a subnotificação de crimes patrimoniais. Assim, adiante são apresentados os resultados da estimação dos modelos econométricos. 


\subsection{Resultados Econométricos}

Conforme destacado na seção anterior, a determinação dos fatores que influenciam a probabilidade de subnotificação de crimes patrimoniais no Brasil em 2009 ocorreu pela estimação de modelos de probabilidade binária (Probit). A validação dos resultados se deu pela estimação da Curva ROC (Receiver Operating Characteristic), que é um teste para indicar o ajustamento dos modelos. De acordo com este método, quanto mais próximo de 1 for a área sob a curva, melhor será a previsão do modelo (BRAGA, 2000; CAMERON e TRIVEDI, 2010; FAVERO e BELFIORI, 2014). Com base nesse procedimento, valores acima de 0,70 já indicam bom ajustamento do modelo. As Figuras 5 e 6 mostram que os modelos apresentaram poder discriminatório satisfatório, visto que os valores calculados sob a Curva ROC foram de 0,7308 e 0,7469 para os modelos estimados para furtos e roubos, respectivamente.

Figura 5 - Curva ROC - Modelo 3 (Furtos)

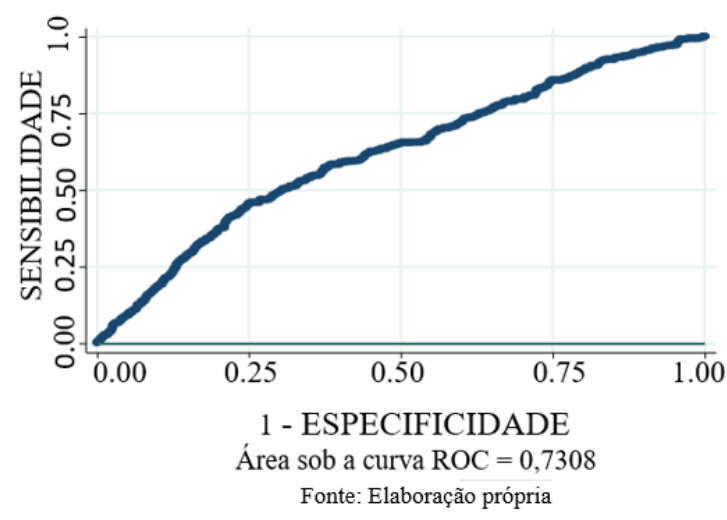

Figura 6 - Curva ROC - Modelo 3 (Roubos)

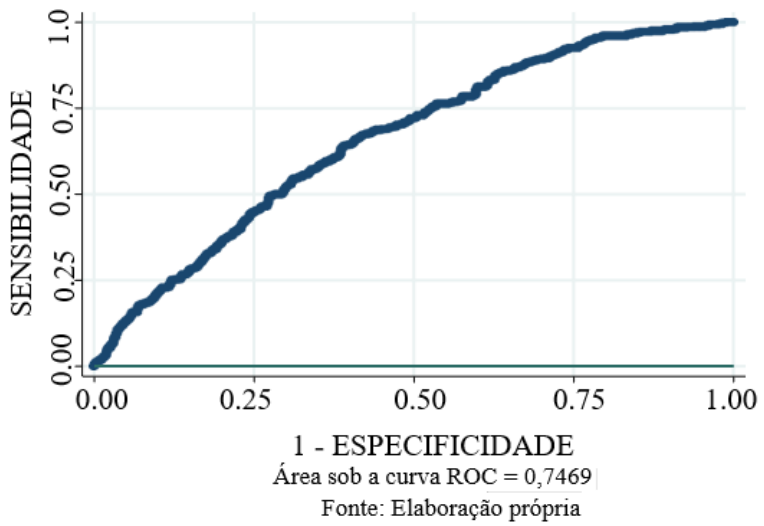


Os resultados da estimação dos modelos, que objetivam elencar os fatores associados à probabilidade de os indivíduos não registrarem os crimes patrimoniais de furtos e roubos, são apresentados nas Tabelas 3 e 4 . A fim de verificar a robustez dos resultados, foram estimados três modelos, inserindo-se de forma gradativa as variáveis de controle. $\mathrm{O}$ primeiro considera apenas escolaridade e renda como variáveis explicativas. No modelo 2, são adicionadas as variáveis sexo, cor, idade, estado civil e ocupação. Por fim, no terceiro modelo são incluídas as variáveis de perdas, região censitária e dummies regionais.

É possível verificar que os coeficientes da variável que denota o nível de renda são estáveis e significativos em todos os modelos estimados. Por sua vez, com relação ao nível de escolaridade, percebe-se que as variáveis que representam o fato de a vítima possuir ensino médio e superior são significativas em todos os modelos de subnotificação de furtos, e não significativas para os modelos de sub-registro de roubos.

Em termos de análise, o Modelo 3, o mais completo, terá seus resultados analisados no presente estudo nas Tabelas 3 e 4. No geral, dentre as variáveis utilizadas no modelo de subnotificação de furtos, vide Tabela 3, apenas as de Ensino Médio, Ensino Superior, Região Nordeste, Renda e Perdas foram estatisticamente significativas. Já para o modelo de subnotificação de roubos, referente à Tabela 4, as variáveis Idade, Região Nordeste, Renda e Perdas apresentaram significância estatística. Além disso, cabe salientar que a maioria das variáveis apresentou sinal esperado.

Conforme observado, para as duas modalidades de crime, as variáveis sexo e cor não foram estatisticamente significativas. Por conseguinte, não é possível afirmar que existem diferenças estatísticas entre homens e mulheres, brancos e não brancos, quanto à decisão de registrar furtos ou roubos. Myers (1980), ao analisar algumas cidades dos Estados Unidos, também não observou efeito de gênero e nem de cor na probabilidade de sub-registro de crimes.

De modo similar, observa-se que as variáveis Estado Civil e a Ocupação do indivíduo não foram estatisticamente significativas. Desse modo, não é possível afirmar que existem diferenças estatísticas nas decisões de um indivíduo solteiro, casado, divorciado ou viúvo em registrar um crime, seja ele de furto ou de roubo. A situação ocupacional do indivíduo também não exerce influência no registro dos referidos crimes. Este resultado está de acordo com os achados de MacDonald (2000) e Duce et. al. (2000), que concluíram que a situação econômica da vítima é um fator mais importante do que o custo de oportunidade de se registrar a ocorrência.

Por outro lado, verifica-se que a idade da vítima se correlaciona negativamente com a probabilidade de subnotificação de roubos. Assim, quanto maior for a idade da vítima, menor será a probabilidade de não registro deste tipo de crime. Duce et al. (2000) corroboram com este resultado. No estudo dos autores, tendo como referência a Espanha, verificou-se que indivíduos mais velhos possuem maior probabilidade de notificar o referido crime à polícia. 
Tabela 3 - Determinantes da Probabilidade de Subnotificação de Furtos no Brasil em 2009

\begin{tabular}{|c|c|c|c|c|c|c|}
\hline \multirow[b]{2}{*}{ Variáveis } & \multicolumn{2}{|c|}{ Modelo 1} & \multicolumn{2}{|c|}{ Modelo 2} & \multicolumn{2}{|c|}{ Modelo 3} \\
\hline & Coeficiente & $\begin{array}{c}\text { Efeitos } \\
\text { Marginais }\end{array}$ & Coeficiente & $\begin{array}{c}\text { Efeitos } \\
\text { Marginais }\end{array}$ & Coeficiente & $\begin{array}{c}\text { Efeitos } \\
\text { Marginais }\end{array}$ \\
\hline Constante & $\begin{array}{c}-0,7424 * * * \\
(0,1678)\end{array}$ & -- & $\begin{array}{l}-0,2361 \\
(0,2292)\end{array}$ & -- & $\begin{array}{l}-0.4591 * \\
(0.2671)\end{array}$ & -- \\
\hline Básico & $\begin{array}{l}-0,0499 \\
(0,1567)\end{array}$ & $-0,0115$ & $\begin{array}{l}-0,0775 \\
(0,1570)\end{array}$ & $-0,0242$ & $\begin{array}{c}-0.0379 \\
(0.1598)\end{array}$ & -0.0104 \\
\hline Fundamental & $\begin{array}{l}-0,2161 \\
(0,1520)\end{array}$ & $-0,0457$ & $\begin{array}{c}-0,2914 * \\
(0,1550)\end{array}$ & $-0,084$ & $\begin{array}{c}-0,2272 \\
(0,1600)\end{array}$ & -0.5729 \\
\hline Médio & $\begin{array}{c}-0,3563 * * \\
(0,1491)\end{array}$ & $-0,0696$ & $\begin{array}{c}-0,4629 * * * \\
(0,1621)\end{array}$ & $-0,1240$ & $\begin{array}{c}-0.3800 * * \\
(0.1678)\end{array}$ & -0.0888 \\
\hline Superior & $\begin{array}{c}-0,4783 \text { *** } \\
(0,1567)\end{array}$ & $-0,0869$ & $\begin{array}{c}-0,5774 * * * \\
(0,1662)\end{array}$ & $-0,1469$ & $\begin{array}{c}-0.4930 * * \\
(0.1704)\end{array}$ & -0.1086 \\
\hline Renda & $\begin{array}{c}-0,039 * * \\
(0,0160)\end{array}$ & $-0,0090$ & $\begin{array}{c}-0,0313^{* *} \\
(0,0142)\end{array}$ & $-0,010$ & $\begin{array}{c}-0.0272 * * \\
(0.0134)\end{array}$ & -0.0076 \\
\hline Sexo & & & $\begin{array}{c}-0,1544 * * \\
(0,06655)\end{array}$ & -0.047 & $\begin{array}{c}-0.1034 \\
(0.0677)\end{array}$ & -0.0276 \\
\hline Cor & & & $\begin{array}{c}-0,750 \\
(0,0636)\end{array}$ & $-0,023$ & $\begin{array}{l}-0.0146 \\
(0.0693)\end{array}$ & -0.0040 \\
\hline Idade & & & $\begin{array}{c}-0,0049 \\
(0,0031)\end{array}$ & $-0,0015$ & $\begin{array}{l}-0.0047 \\
(0.0031)\end{array}$ & -0.0013 \\
\hline Estado Civil & & & $\begin{array}{c}-0,0258 \\
(0,0694)\end{array}$ & $-0,0082$ & $\begin{array}{c}-0.0927 \\
(0.0710)\end{array}$ & -0.0080 \\
\hline Ocupação & & & $\begin{array}{l}-0,1404 \\
(0,1303)\end{array}$ & $-0,0429$ & $\begin{array}{l}-0.1125 \\
(0.1317)\end{array}$ & -0.0299 \\
\hline $\begin{array}{c}\text { Região } \\
\text { Censitária }\end{array}$ & & & & & $\begin{array}{c}0.0275 \\
(0.1243) \\
\end{array}$ & 0.0077 \\
\hline Nordeste & & & & & $\begin{array}{l}0.2589^{*} \\
(0.1208)\end{array}$ & 0.0798 \\
\hline $\begin{array}{l}\text { Centro } \\
\text { Oeste }\end{array}$ & & & & & $\begin{array}{r}0.1930 \\
(0.1291) \\
\end{array}$ & 0.0581 \\
\hline Sudeste & & & & & $\begin{array}{l}-0.0413 \\
(0.1205)\end{array}$ & -0.0113 \\
\hline Sul & & & & & $\begin{array}{l}-0.0705 \\
(0.1324)\end{array}$ & -0.0191 \\
\hline Perdas & & & & & $\begin{array}{c}-0.5214 * * * \\
(0.1492)\end{array}$ & -0.1132 \\
\hline
\end{tabular}

Fonte: Elaboração própria. Observações $=3898$

Nota: (a) *** $\mathrm{p}<0.01, * * \mathrm{p}<0.05$, *p<0.1; erro padrão entre parênteses;

(b) A faixa de um ou menos anos de estudo é omitida dentre as dummies de escolaridade.

(c) O peso amostral foi considerado nas estimações. 
Tabela 4 - Determinantes da probabilidade de subnotificação de roubos no Brasil em 2009

\begin{tabular}{|c|c|c|c|c|c|c|}
\hline \multirow[b]{2}{*}{ Variáveis } & \multicolumn{2}{|c|}{ Modelo 1} & \multicolumn{2}{|c|}{ Modelo 2} & \multicolumn{2}{|c|}{ Modelo 3} \\
\hline & Coeficiente & $\begin{array}{c}\text { Efeitos } \\
\text { Marginais }\end{array}$ & Coeficiente & $\begin{array}{c}\text { Efeitos } \\
\text { Marginais }\end{array}$ & Coeficiente & $\begin{array}{c}\text { Efeitos } \\
\text { Marginais }\end{array}$ \\
\hline Constante & $\begin{array}{c}-1,0750 * * * \\
(0,1498) \\
\end{array}$ & -- & $\begin{array}{c}-0,6824 * * * \\
(0,2154) \\
\end{array}$ & -- & $\begin{array}{c}-0.9059 * * * \\
(0.2704) \\
\end{array}$ & -- \\
\hline Básico & $\begin{array}{c}-0,0941 \\
(0,1580)\end{array}$ & 0,0173 & $\begin{array}{c}0,0878 \\
(0,1621)\end{array}$ & 0,0194 & $\begin{array}{l}-0.1400 \\
(0.1630)\end{array}$ & 0.0269 \\
\hline Fundamental & $\begin{array}{l}-0,1932 \\
(0,1435)\end{array}$ & $-0,0295$ & $\begin{array}{c}-0,2527 * \\
(0,1498)\end{array}$ & $-0,0458$ & $\begin{array}{l}-0,1713 \\
(0,1523)\end{array}$ & $-0,0270$ \\
\hline Médio & $\begin{array}{c}-0,0481 \\
(0,1419)\end{array}$ & $-0,0081$ & $\begin{array}{c}-0,1559 \\
(0,1525)\end{array}$ & $-0,0300$ & $\begin{array}{c}-0.0568 \\
(0.1560)\end{array}$ & -0.0096 \\
\hline Superior & $\begin{array}{c}-0,1618 \\
(0,1490) \\
\end{array}$ & $-0,0253$ & $\begin{array}{c}-0,2403 \\
(0,1598)\end{array}$ & $-0,0439$ & $\begin{array}{l}-0.1115 \\
(0.1646) \\
\end{array}$ & -0.0183 \\
\hline Renda & $\begin{array}{c}-0,0286^{* * *} \\
(0,0090)\end{array}$ & $-0,0049$ & $\begin{array}{c}-0,2509 * * * \\
(0,0085)\end{array}$ & $-0,0052$ & $\begin{array}{c}-0.0216^{* *} \\
(0.0082) \\
\end{array}$ & -0.0038 \\
\hline Sexo & & & $\begin{array}{c}-0,1312 * * \\
(0,0589)\end{array}$ & $-0,0256$ & $\begin{array}{c}-0.0787 \\
(0.0605) \\
\end{array}$ & -0.0132 \\
\hline Cor & & & $\begin{array}{c}-0,1463 * * \\
(0,0635)\end{array}$ & $-0,0283$ & $\begin{array}{c}-0.0699 \\
(0.0628)\end{array}$ & -0.0118 \\
\hline Idade & & & $\begin{array}{c}-0,0071 * * \\
(0,0027)\end{array}$ & $-0,0015$ & $\begin{array}{c}-0.0057 * * \\
(0.0028)\end{array}$ & -0.0010 \\
\hline Estado Civil & & & $\begin{array}{l}-0,0518 \\
(0,0623) \\
\end{array}$ & $-0,0106$ & $\begin{array}{l}-0.0571 \\
(0.1623) \\
\end{array}$ & -0.0097 \\
\hline Ocupação & & & $\begin{array}{c}0,088 \\
(0,1011)\end{array}$ & 0,0194 & $\begin{array}{c}0.1172 \\
(0,1021)\end{array}$ & 0.0222 \\
\hline $\begin{array}{c}\text { Região } \\
\text { Censitária }\end{array}$ & & & & & $\begin{array}{c}-0.0945 \\
(0.1464)\end{array}$ & -0.0156 \\
\hline Nordeste & & & & & $\begin{array}{c}0.2850 * * \\
(0.0977)\end{array}$ & 0.0598 \\
\hline Centro Oeste & & & & & $\begin{array}{c}0.1098 \\
(0.1187) \\
\end{array}$ & 0.0207 \\
\hline Sudeste & & & & & $\begin{array}{c}0.0678 \\
(0.1030) \\
\end{array}$ & 0.01249 \\
\hline Sul & & & & & $\begin{array}{l}0.00129 \\
(0.1254)\end{array}$ & 0.0002 \\
\hline Perdas & & & & & $\begin{array}{c}-0.6414 * * * \\
(0.2704)\end{array}$ & -0.0732 \\
\hline
\end{tabular}

Fonte: Elaboração própria. Observações $=5312$

Nota: (a) *** $\mathrm{p}<0.01, * * \mathrm{p}<0.05, * \mathrm{p}<0.1$; erro padrão entre parênteses;

(b) A faixa de um ou menos anos de estudo é omitida dentre as dummies de escolaridade;

(c) $\mathrm{O}$ peso amostral foi considerado nas estimações. 
Em relação à escolaridade dos indivíduos, é possível observar que aqueles com mais anos de estudo apresentaram maior probabilidade de registrar a ocorrência de furtos. Tal qual apresentado na Tabela 3, os indivíduos com ensino médio e superior têm probabilidade mais elevada de registrar um furto comparativamente aos analfabetos. Consequentemente, constata-se que existe relação negativa entre a probabilidade de subnotificação do referido crime e o fato de o indivíduo possuir ensino médio/superior. Isto posto, destaca-se que o fato de a vítima possuir ensino médio diminui sua probabilidade em 0,088 ponto percentual. Já o fato de ter ensino superior reduz em 0,1 ponto percentual a probabilidade de sub-registro de furtos, em relação aos analfabetos. Duce et al. (2000) corroboram com este resultado, ao salientarem que as pessoas com maior nível de escolaridade são mais propensas a registrar crimes do que pessoas com menos anos de estudos. Em contrapartida a estes resultados, observa-se que não há relação entre o nível de escolaridade da vítima com a probabilidade de não se registrar um roubo. Myers (1980) reforça tal resultado ao afirmar que a educação não explica qualquer variação na probabilidade de sub-registro de crimes entres as cidades dos Estados Unidos.

Com relação à questão localizacional, o fato de o indivíduo residir nas zonas urbana ou rural não está relacionado à probabilidade de registro de furtos e roubos. Ademais, dentre as regiões brasileiras, observa-se que existem diferenças estatísticas entre a probabilidade de subnotificação dos crimes de furto e roubo apenas entre as regiões Norte (região de referência) e Nordeste. Conforme apresentado nas Tabela 3 e 4, destaca-se que a região nordestina tende a apresentar maior probabilidade de não registro de um crime. Tal resultado condiz com a análise descritiva apresentadas na subseção anterior, onde observou-se que a região Nordeste do Brasil é aquela com maior índice de subnotificação dos crimes de furtos e roubos. Assim, residir no Nordeste aumenta a probabilidade de sub-registro em 0,079 ponto percentual, nos casos de furto ,e 0,05 ponto percentual, nos casos de roubo. Barros et al. (2001) ressaltam ainda que os indivíduos desta região apresentam menor nível de escolaridade, o que aumenta a probabilidade destes não registarem o crime sofrido.

Quanto à variável renda, salienta-se que esta se correlaciona negativamente com a probabilidade de incidência de subnotificação de crimes patrimoniais. Dito isso, infere-se que um adicional de $\mathrm{R} \$ 100,00$ na renda da vítima reduzirá a probabilidade de subnotificação para furtos e roubos em 0,007 e 0,003 ponto percentual, respectivamente. O resultado encontrado diverge daqueles verificados por Myers (1980) e Golderbeg e Nold (1980), os quais não observam coeficientes estatisticamente significativos para a variável que reflete a renda da vítima. Entretanto, o referido resultado está de acordo com o aquele encontrado por Santos e Kassouf (2008), onde os autores observam relação positiva entre as variáveis de renda e a probabilidade de se registrar um crime.

Por fim, a variável Perdas, incluída no modelo para captar o efeito da análise de custo-benefício da vítima sobre a decisão de se registrar a vitimização ou não, apresentou-se estatisticamente significativa em ambos os casos. Por conseguinte, este 
resultado confirma a hipótese de que maiores perdas materiais levam à maior probabilidade de se registrar um crime.

\section{Considerações Finais}

A existência de elevados índices de subnotificação de crimes é um problema tanto de ordem social quando econômico. Do ponto de vista social, o sub-registro dos crimes expressa a crença de ineficiência e baixo nível de credibilidade da segurança pública. Em termos econômicos, a subnotificação conduz a uma alocação ineficiente dos recursos públicos, visto que estes não serão empregados, necessariamente, onde há maior necessidade. Posto isto, reduzir a subnotificação dos crimes, especificamente de furtos e roubos contra o patrimônio, torna-se objeto de suma importância para a esfera pública.

Assim, este estudo teve como objetivo analisar os determinantes da probabilidade de subnotificação de crimes contra o patrimônio no Brasil em 2009. Conforme observado nos resultados, no geral, os principais fatores determinantes da probabilidade de as vítimas não registrarem os crimes sofridos, e considerados no trabalho, foram: idade; nível de escolaridade; renda; região; e valor das perdas. Destes, as variáveis que representam a região Nordeste e o baixo nível de escolaridade se apresentaram positivamente correlacionados com a subnotificação. Por outro lado, indivíduos com maior nível de escolaridade, renda e idade tendem a apresentar menor probabilidade de não registrar um crime sofrido.

É importante ressaltar que registrar um crime é a base para que o responsável seja punido e, consequentemente, para que haja maior probabilidade de insucesso da atividade criminosa, o que implicaria em menor predisposição à criminalidade. Assim, observa-se a importância de políticas que tenham como objetivo elevar o nível de conscientização da população e confiabilidade na eficiência da polícia, particularmente entre indivíduos com menor nível de escolaridade e renda residentes na região Nordeste, assim como para aqueles cujas perdas monetárias foram relativamente menores.

Por fim, cabe ressaltar algumas limitações do presente estudo. A base de dados utilizada corresponde a uma pesquisa de vitimização realizada pela PNAD, que possui algumas desvantagens, tais como a falta de memória do entrevistado, particularmente quando questionado a respeito de ter sofrido um determinado crime a algum tempo; a realização desta em domicílios, de modo que a pessoa de referência responde pelos outros; e a possibilidade de a informação fornecida pelo entrevistado ser falsa. Além disso, o modelo utilizado não permite o controle da possível relação endógena entre as variáveis dependentes e algumas explicativas. Isso poderia ser feito utilizando-se variáveis instrumentais, que em uma análise cross-section como esta é dificultada pela inexistência instrumentos adequados. 


\section{Referências}

ANDRADE, M. V.; LISBOA, M. B. Desesperança de vida: Homicídio em Minas Gerais. In Henriques, R. Desigualdade e Pobreza no Brasil, p. 347-384. IPEA, Rio de Janeiro.2000.

JÚNIOR, A.A.; FAJNZYLBER, P. O que causa a criminalidade violenta no Brasil? Uma análise a partir do modelo econômico do crime: 1981 a 1996. Texto de Discussão, n.162. Universidade Federal de Minas Gerais, CEDEPLAR, set. 2001.

BARROS, R. P. D.; MENDONÇA, R.; SANTOS, D. D. D.; QUINTAES, G. Determinantes do Desempenho Educacional no Brasil. 2001.

BECKER, G. S. Irrational behavior and economic theory. The Journal of Political Economy, Chicago, v.70, n. 1, p. 1-13, Feb. 1962.

DOI: https://doi.org/10.1086/258584

BECKER, G. S. Crime and punishment: an economic approach. Journal of Political Economy, v. 76, n. 2, p. 169-217, 1968.

DOI: https://doi.org/10.1086/259394

BRAGA, A. C. S. Curvas ROC, Aspectos Funcionais e Aplicações. Tese de Doutorado. Dissertação (Doutorado em Engenharia de Produção e Sistemas) - Área de métodos numéricos e estatísticos, Universidade do Minho. 2000.

CAMERON, A. C.; TRIVEDI, P. K. Microeconometrics Using Stata. Rev. ed. College Station, TX: Stata Press. 2010.

CRAIG, S. G. The Deterrent Impact of Police: An Examination of a Locally Provided Public Service. Journal of Urban Economics, v.21, p.298-311, 1987.

DOI: https://doi.org/10.1016/0094-1190(87)90004-0

DUCE, A. D. T.; CHAVARRÍA, P. L.; TORRUBIA, M. J. M. Análisis Microeconómico de los Datos Criminales: factores determinantes de la probabilidade de denunciar um delito. 2000.

FAJNZYLBER, P.; JÚNIOR, A.A. Violência e Criminalidade. Texto para Discussão. Belo Horizonte: UFMG, p. 1-50, 2001.

FÁVERO, L. P.; BELFIORE P. Métodos Quantitativos com Stata. Rio de Janeiro: Elsevier. 248 p. 2014.

GOLDEBERG, G. E.; NOLD, F. C. Does reporting deter burglars? An empirical analysis of risk and return in crime. Review of Economics and Statistics, V. 62, N.3, p. 424-431, 1980. DOI: https://doi.org/10.2307/1927110 
GOUDRIAAN, H.; ASSEN, G. T. Microeconometrics Using Stata. Veenendaal: Universal Press. 212 p. 2006.

MACDONALD, Z. Official Crime Statistics: Their Use and Interpretation. The Economic Journal, Leicester: v. 112, n. 477, 2002. p. 85-106.

DOI: https://doi.org/10.1111/1468-0297.00685

MADALOZZO, R.; FURTADO, G. Um estudo sobre a vitimização para a cidade de São Paulo, Revista de Economia Política, v.31, n.1, p. 160-180, 2011.

DOI: https://doi.org/10.1590/S0101-31572011000100008

MYERS, S. L. Jr. Why are crimes underreported? What is the crime rate? Does it really matter? Social Science Quarterly, Austin,v. 61, n. 1, p. 23-43, June 1980.

OLIVEIRA, C. A. Criminalidade e o tamanho das cidades brasileiras: Um enfoque da economia do crime. In XXXIII Encontro Nacional de Economia. ANPEC, Natal. 2005. Disponível em: <http://econpapers.repec.org/paper/anpen2005/152.htm>

REICHEL, P.; ALBANESE, J. Handbook of Transnational Crime and Justice. SAGE Publications, Inc., 2014.

SANTOS, M. J.; KASSOUF, A. L. Existe Explicação Econômica para o Sub-Registro de Crimes Contra a Propriedade? Economia Aplicada, São Paulo, v.12, n.1, p.5-27, jan/mar., 2008.

DOI: https://doi.org/10.1590/S1413-80502008000100001

SCORZAFAVE, L.; SANTOS, M.; KASSOUF, A. Determinantes da vitimização e do sub-registro de crimes na cidade de São Paulo, Relatório de Pesquisa, CPP-Insper, 2011. 